\title{
Abordaje del desarrollo en la primera infancia y sus dificultades en Turquía: programa de capacitación para las unidades de pediatría del desarrollo

\author{
Addressing early childhood development and developmental \\ difficulties in Turkey: a training program for developmental \\ pediatrics units
}

Medicina de Ankara

Üniversitesi,

Departamento de

Pediatría, División

de Pediatría del

Desarrollo y el Comportamiento,

Ankara, Turquía.

b. Hospital de

Formación e

Investigación en

Salud Infantil y

Enfermedades

Pediátricas,

Hematología

y Oncología,

Universidad de

Ciencias de la Salud,

Ankara, Turquía.

c. Hospital de

Formación e

Investigación Şişli

Hamidiye Etfal,

Universidad de

Ciencias de la Salud,

Estambul, Turquía.

d. Hospital de

Formación e

Investigación

Materno Infantil

Dr. Sami Ulus,

Ankara, Turquía.

e. Hospital Pediátrico

de Formación e

Investigación

Dr. Behçet Uz,

Esmirna, Turquía.

f. Hospital de

Formación e

Investigación en

Salud de la Mujer

Zekai Tahir Burak,

Universidad de

Ciencias de la Salud,

Ankara, Turquía.

Correspondencia:

Dra. Emine B. Bingoler Pekcici:

baharbingoler@yahoo. com

\author{
Dra. Emine B. Bingoler Pekcici ${ }^{a}$ Dra. Ezgi Özalp Akin ${ }^{a}$ Dra. Iclal Ayranci Sucaklib, \\ Dra. Gizem Kara Elitokc, Dra. Fatma N. Onat ${ }^{d}$, Dra. Meltem Kivilcim ${ }^{e}$, \\ Dra. Zeynep Ustunyurt, Dr. Revan Mustafayev y Dra. Ilgi Ozturk Ertem ${ }^{a}$
}

\section{RESUMEN}

Introducción. El abordaje del desarrollo en la primera infancia (DPI) y las dificultades del desarrollo (DD) en los países de ingresos bajos o medios (PIBM) requiere de programas aplicables y sostenibles. Se evaluó el programa de capacitación para las unidades de pediatría del desarrollo (UPD) en los centros de referencia tras siete años.

Población y métodos. Diseño transversal con métodos cualicuantitativos para evaluar los servicios, la capacitación, la investigación y la promoción de las UPD.

Resultados. Semantuvieron los servicios clínicos, la capacitación, investigación y promoción. En total, 23710niños fueronderivados. La motivación y resistencia del personal, el respaldo de los administradores y la satisfacción del paciente facilitaron el programa, aunque la invisibilidad dentro del sistema de salud fue un obstáculo. Conclusiones. En Turquía, y posiblemente otros PIBM, el programa de capacitación para las UPD es aplicable y sostenible si se abordan los obstáculos del sistema de salud.

Palabras clave: desarrollo infantil, niños con discapacidad, población de ingresos bajos.

http: / / dx.doi.org/10.5546/ aap.2020.e384

Texto completo en inglés:

http: / / dx.doi.org/10.5546/ aap.2020.eng.e384

Cómo citar: Bingoler Pekcici EB, Özalp Akin E, Ayranci Sucakli I, Kara Elitok G, et al. Abordaje del desarrollo en la primera infancia y sus dificultades en Turquía: programa de capacitación para las unidades de pediatría del desarrollo. Arch Argent Pediatr 2020;118(4):e384-e391.

\section{INTRODUCCIÓN}

Recientemente, la Organización Mundial de la Salud (OMS) publicó el Marco para el cuidado cariñoso y sensible para el desarrollo en la primera infancia, en el cual se definen las "dificultades del desarrollo" (DD) como cualquier condición que ponga a un niño en riesgo de un desarrollo subóptimo o que cause una desviación, un retraso, un trastorno o una discapacidad del desarrollo. El término abarca a todos los niños con limitaciones en el funcionamiento y el desarrollo de su máximo potencial. ${ }^{1}$ Los Objetivos de Desarrollo Sostenible 2030 de las Naciones Unidas incluyen acceso al desarrollo en la primera infancia (DPI) de calidad para todos los niños, con énfasis en la importancia de abordar el DPI y las DD en el marcode los sistemas de salud. ${ }^{2}$ La mayoría de los niños, y el $95 \%$ de aquellos con $\mathrm{DD}$, viven en países de ingresos bajos y medios (PIBM). ${ }^{3}$ La mortalidad neonatal e infantil, el foco anterior de los sistemas de salud dentro de los PIBM, ha disminuido, ${ }^{4} \mathrm{y}$ se requieren cada vez más estrategias para abordar el DPI y las DD. ${ }^{2}$ Dos décadas atrás, el documento de estrategia trascendental titulado Neurological, Psychiatric, and Developmental Disorders: Meeting the Challenge in the Developing World destacó la importancia de fortalecer los centros de referencia para abordar las DD. ${ }^{5}$ Si bien se dispone de cierta 
información sobre cómo pueden los sistemas de atención primaria de la salud abordar el DPI, ${ }^{6}$ las investigaciones sobre cómo abordar las DD en los centros de referencia en los PIBM son escasas. ${ }^{6}$

Las investigaciones de varios países de ingresos altos (PIA), como Australia, ${ }^{7}$ Israel $^{8} \mathrm{y}$ los Estados Unidos, ${ }^{9}$ sugieren que los pediatras requieren capacitación para abordar el DPI y las DD. En estos países, esta capacitación se basa en la pediatría del desarrollo y el comportamiento (PDC) o en las rotaciones de psiquiatría pediátrica, o en ambas. ${ }^{10}$ Un obstáculo importante para los pediatras en los PIBM ha sido la falta de oportunidades para recibir dicha capacitación. ${ }^{11,12}$ Las variaciones en los factores de riesgo del desarrollo ${ }^{2}$ y en el conocimiento de los cuidadores ${ }^{13} \mathrm{y}$ los médicos ${ }^{14}$ podrían requerir una capacitación que ataña específicamente a los PIBM. Asimismo, los equipos multidisciplinarios capaces de trabajar en conjunto para llevar a cabo evaluaciones integrales e intervenciones en los PIA podrían, a menudo, no estar disponibles en los centros de referencia de los PIBM. ${ }^{12}$

A fin de abordar la necesidad de programas de capacitación, entre 2005 y 2010, se desarrolló un paquete de capacitación denominado "Abordaje de las dificultades del desarrollo y del desarrollo en la primera infancia (ADD+DPI)", que apunta a mejorar el conocimiento, las habilidades y las actitudes de los profesionales sanitarios en los centros de atención primaria y de referencia para el monitoreo y el respaldo del DPI, y la prevención, la identificación temprana y el manejo de las DD. ${ }^{15}$ Se brindó capacitación a los médicos de más de 30 países sobre varios componentes del ADD+DPI, en particular, la Guía internacional para el monitoreo del desarrollo infantil (GMDI), una herramienta universal para la prevención e identificación temprana de las DD y la intervención temprana. ${ }^{14,16-18}$ Previamente, habíamos presentado un informe sobre uno de los componentes del ADD+DPI, el programa de capacitación de tres días para los profesionales de la atención primaria de la salud, que incluyó brindar asesoramiento sobre el desarrollo infantil a los cuidadores, monitorear el desarrollo para una identificación temprana y el manejo de las DD durante las consultas médicas de rutina. Nuestra investigación cualitativa sobre este programa en Turquía reveló que las consultas en los centros de referencia con profesionales sanitarios capacitados no eran eficaces. ${ }^{14}$ Los centros de referencia no contaban con pediatras que pudieran hacer evaluaciones integrales y brindar atención a los niños con DD ni tenían un espacio específico donde estos niños pudieran recibir atención. ${ }^{14}$ Ahora informamos sobre el segundo componente del ADD+DPI, el programa para las unidades pediátricas del desarrollo (UPD). A diferencia de la capacitación para el entorno de la atención primaria, el programa para las UPD está diseñado para satisfacer las necesidades de los centros pediátricos de referencia y apunta a capacitar a un equipo multidisciplinario de pediatras y médicos de disciplinas aliadas para que puedan establecer una UPD dentro del centro de referencia. El objetivo de este estudio fue evaluar, siete años después de la implementación de las UPD, los servicios clínicos, la capacitación, la investigación y la promoción resultantes del programa para las UPD, además de los catalizadores y obstáculos de los servicios de las UPD.

\section{POBLACIÓN Y MÉTODOS El programa para las UPD}

Los objetivos del programa para las UPD, de tres meses de duración, fueron brindar a los participantes una actualización de los conocimientos, las habilidades y las actitudes en relación con el abordaje del DPI y las DD y fomentar su capacidad para formar y mantener una UPD en el centro de referencia. Estos objetivos incluyeron mejorar las habilidades para lo siguiente: a) brindar servicios clínicos para promocionar el DPI, reconocer los factores de riesgo del desarrollo, hacer evaluaciones del desarrollo integrales y centradas en la familia, usar herramientas de evaluación estandarizadas, manejar las DD con un plan individualizado de intervención temprana y coordinar los servicios; b) capacitar a los residentes de pediatría, los médicos de cabecera y otros miembros del personal sanitario; c) investigar y d) promover el DPI y las DD. Una de las principales metas del programa era fomentar el entusiasmo y la devoción persistentes de los participantes de la capacitación para aprender y prestar servicios a los niños y sus cuidadores con humildad.

Uno de los autores (IE) preparó el plan de capacitación para las UPD sobre la base de los planes de estudio existentes de los programas sobre DPI y DD e incorporó las experiencias a largo plazo para así satisfacer las necesidades en Turquía y, potencialmente, otros PIBM. Sobre la base de la teoría bioecológica del desarrollo infantil ${ }^{19}$ y enfoques centrados en la familia, basados en las fortalezas y las relaciones, ${ }^{20} \mathrm{el}$ 
marco de la Clasificación internacional del funcionamiento, de la discapacidad y de la salud (CIF) de la OMS ${ }^{21}$ y los principios transdisciplinarios, ${ }^{22}$ se llevaron a cabo sesiones teóricas (180 horas) y prácticas (240 horas). En la Tabla 1, se incluye una lista completa de los seminarios teóricos. Las referencias incluyeron un manual desarrollado específicamente para la capacitación, el manual de referencia "Pediatría del desarrollo" en turco, artículos, libros, revistas, diapositivas de PowerPoint y videos educativos.

Los objetivos de las sesiones prácticas fueron permitir que los médicos hicieran evaluaciones del desarrollo transdisciplinarias mediante métodos centrados en la familia que permitieran realizar un diagnóstico, siempre que fuera posible, y un plan de intervención temprana integral basado en el marco de la CIF (estructuras corporales, funciones, actividades y participación en la vida) para satisfacer las necesidades de los niños y sus familias. Los sistemas de clasificación diagnóstica empleados incluyeron la CIF de la OMS, el Manual diagnóstico y estadístico de los trastornos mentales, $4 .^{\text {a }}$ edición (DSM-IV), y la Clasificación diagnóstica de cero a tres de los trastornos del desarrollo y la salud mental durante la lactancia y la primera infancia (DC:0-3). Las herramientas utilizadas incluyeron la GMDI; la GMDI ampliada, que abarca todos los dominios de la CIF; las Escalas conductuales adaptativas de Vineland, segunda edición; las Escalas Bayley de desarrollo de lactantes y niños pequeños, segunda edición; las Observaciones del hogar para la medición del ambiente; la Lista de verificación modificada del autismo en niños pequeños; y mediciones de funcionamiento y salud mental familiar. La capacitación incluyó hacer derivaciones eficientes y eficaces para la realización de pruebas específicas (por ejemplo, pruebas auditivas, visuales y del lenguaje) o para tratamientos específicos (por ejemplo, parálisis cerebral, autismo, problemas de salud mental de los cuidadores), siempre que fueran necesarios. La práctica implicó la observación de los niños y sus familias en entornos de la vida real, como hogares, hospitales, establecimientos preescolares y centros de intervención temprana. Los participantes observaron a los mentores con antecedentes en pediatría del desarrollo y

TABLA 1. Temas teóricos de los seminarios del programa para las unidades pediátricas del desarrollo

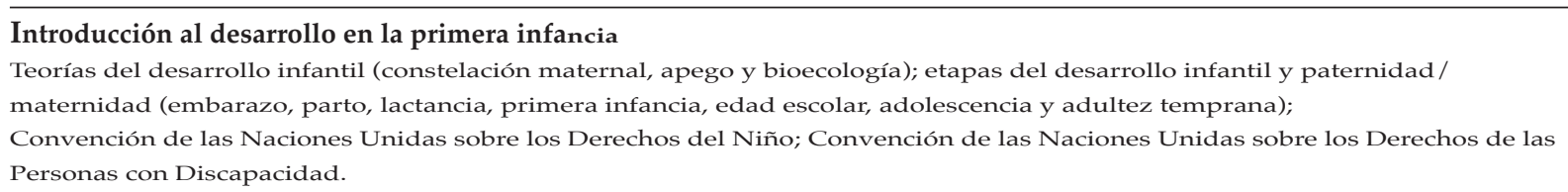

Factores de riesgo del desarrollo y resiliencia

Factores de riesgo psicológicos (pobreza, estrés, depresión parental, abuso y abandono, abuso de sustancias, situaciones de crisis y catástrofes); factores de riesgo biológicos (desnutrición, anemia, ferropenia, factores de riesgo perinatales, como prematurez, genéticos, neurológicos, trastornos endocrinos/metabólicos, otras enfermedades crónicas y sus efectos en el desarrollo); abordaje de los factores de riesgo y supervisión y respaldo del desarrollo durante la lactancia, primera infancia, edad escolar, adolescencia y adultez temprana.

Principios de las evaluaciones del desarrollo integrales y centradas en la familia

Anamnesis del desarrollo; técnicas de observación del desarrollo; selección de instrumentos según los distintos grupos etarios y contextos; evaluación del funcionamiento, las actividades y la participación del niño, el contexto familiar y comunitario, los servicios y sistemas; proceso de evaluación como una intervención, con una transición uniforme hacia una intervención temprana.

Sistemas de clasificación diagnóstica

Clasificación internacional del funcionamiento, de la discapacidad y de la salud de la OMS; Manual diagnóstico y estadístico de los trastornos mentales; Clasificación diagnóstica de cero a tres de los trastornos del desarrollo y la salud mental durante la lactancia y la primera infancia.

Evaluación, diagnósticos, tratamiento, rehabilitación, gestión de casos y seguimiento de trastornos del desarrollo específicos Retraso del lenguaje, trastorno del espectro autista, parálisis cerebral, trastornos del sistema nervioso central y periférico, deterioros de la función cognitiva, del aprendizaje, del habla, visuales, auditivos y múltiples; trastorno conductual de la alimentación, trastornos del sueño, enuresis, encopresis.

Programas y sistemas para respaldar el desarrollo

La función del sistema de salud, programas basados en el hogar y la familia, visitas al hogar, programas comunitarios, programas basados en centros de rehabilitación, enfoques transdisciplinarios, promoción eficaz y desarrollo de programas en el DPI. 
el comportamiento y en psiquiatría y psicología pediátricas antes de hacer las evaluaciones e intervenciones. Mediante la observación directa de los mentores o la grabación en video, fue posible hacer una supervisión reflexiva. Los participantes, bajo supervisión, evaluaron y brindaron seguimiento a, por lo menos, 50 niños con diferentes DD.

La capacitación incluyó un curso de ocho horas de duración sobre metodología de investigación. Los proyectos de promoción correspondieron a mejorar las prácticas dentro del sistema de salud para fomentar el DPI y las DD, hacer peticiones, presentarse en el parlamento y los ministerios para solicitar cambios legislativos y trabajar con organizaciones sin fines de lucro para cumplir con las demandas de los niños con necesidades especiales.

El programa para las UPD se implementó en Turquía en 2010 como modelo de cooperación multilateral entre el ámbito académico, los centros pediátricos de referencia, el Ministerio de Salud y el Fondo Internacional de Emergencia de las Naciones Unidas para la Infancia (UNICEF). Toda la capacitación estuvo a cargo de la Facultad de Medicina de Ankara Üniversitesi (AU), Departamento de Pediatría, División de Pediatría del Desarrollo y el Comportamiento. Por medio de AU, se dispuso de un profesor a tiempo completo y otros cinco académicos que trabajaron dos horas cada uno. La administración de los centros de referencia designó una sala para la UPD; el Ministerio de Salud cubrió el salario del personal; y UNICEF financió los viajes y el alojamiento de los participantes que vivían fuera de Ankara y las herramientas del desarrollo para las UPD. Entre 59 hospitales similares del país, el Ministerio de Salud seleccionó aquellos con la mayor cantidad de camas y residentes de pediatría, y la administración del hospital eligió a los participantes del programa para las UPD. Antes de la implementación del programa, los participantes no trabajaban en equipo y no habían recibido capacitación previa en DPI ni DD. Los pediatras y médicos de otras disciplinas aliadas (psicólogos, educadores especiales) cumplían los requisitos pero, debido al personal insuficiente, se inscribió solo a los pediatras y especialistas en desarrollo infantil. Los criterios de selección incluyeron interés en el DPI y las DD, ofrecerse como voluntario para la capacitación de tres meses, ser considerado como médico líder por parte de la administración y trabajar bien con los niños y sus familias.

\section{Diseño y mediciones del estudio}

En este estudio transversal, se aplicaron métodos cualicuantitativos. Los resultados del programa para las UPD se evaluaron mediante una encuesta desarrollada especialmente que incluía preguntas estructuradas y abiertas sobre las características de los centros de referencia y

TABla 2. Motivos de derivación a las unidades de pediatría del desarrollo

\begin{tabular}{|c|c|c|c|c|c|c|c|c|c|c|}
\hline \multirow[t]{2}{*}{ Motivos de derivación ( $N=23$ 710) } & \multicolumn{2}{|c|}{ UPD-1 } & \multicolumn{2}{|c|}{ UPD-2 } & \multicolumn{2}{|c|}{ UPD-3 } & \multicolumn{2}{|c|}{ UPD-4 } & \multicolumn{2}{|c|}{ UPD-5 } \\
\hline & n & $(\%)$ & n & $(\%)$ & n & $(\%)$ & n & $(\%)$ & n & $(\%)$ \\
\hline Seguimiento de prematuros y problemas perinatales & 696 & 44,8 & 1130 & 35,3 & 3094 & 46,4 & 436 & 33,4 & 5214 & 80,0 \\
\hline $\begin{array}{l}\text { Afecciones crónicas del desarrollo (trastornos congénitos, } \\
\text { genéticos, metabólicos y neurológicos, trastorno del } \\
\text { espectro autista, parálisis cerebral, niños en guarda) }\end{array}$ & 1534 & 25,5 & 871 & 27,2 & 1614 & 24,2 & 253 & 19,4 & 886 & 13,6 \\
\hline Retraso del habla y el lenguaje & 1089 & 18,1 & 714 & 22,3 & 733 & 11,0 & 227 & 17,4 & 267 & 4,1 \\
\hline Problemas conductuales & 385 & 6,4 & 195 & 6,1 & 640 & 9,6 & 109 & 8,3 & 104 & 1,6 \\
\hline Deficiencias visuales y auditivas & 18 & 0,3 & 0 & 0 & 13 & 0,2 & 9 & 0,7 & 46 & 0,7 \\
\hline Otros trastornos del desarrollo & 90 & 1,5 & 0 & 0 & 287 & 4,3 & 0 & 0 & 0 & 0 \\
\hline $\begin{array}{l}\text { Niños derivados por retraso del desarrollo pero } \\
\text { identificados como que tenían desarrollo típico }\end{array}$ & 205 & 3,4 & 292 & 9,1 & 287 & 4,3 & 272 & 20,8 & 0 & 0 \\
\hline Total & 6017 & 100 & 3202 & 100 & 6668 & 100 & 1306 & 100 & 6517 & 100 \\
\hline
\end{tabular}

UPD-1: Ministerio de Salud de Turquía (MST), Hospital de Formación e Investigación en Salud Infantil y Enfermedades Pediátricas, Hematología y Oncología, Ankara; UPD-2: MST, Hospital Pediátrico de Formación e Investigación Dr. Behçet Uz, Esmirna; UPD-3: MST, Hospital de Formación e Investigación Materno Infantil Dr. Sami Ulus, Ankara; UPD-4: MST, Hospital de Formación e Investigación Şişli Hamidiye Etfal, Estambul; UPD-5: MST, Hospital de Formación e Investigación en Salud de la Mujer Zekai Tahir Burak, Ankara. 
los servicios provistos en las UPD. Los pediatras de las UPD completaron la encuesta en línea con la herramienta SurveyMonkey en enero de 2018. Para obtener información cualitativa adicional, dos de los autores (BBP e IE) entrevistaron personalmente al personal de las UPD y a los administradores de los hospitales donde se encontraban las UPD. El estudio fue aprobado por los comités de ética de AU y de todas las instituciones donde funcionaban las UPD.

\section{Análisis de los datos}

Se utilizaron estadísticas descriptivas para analizar la cantidad de médicos capacitados y los servicios prestados. Se hizo la prueba z para grupos independientes para determinar si las proporciones de los motivos de las derivaciones eran estadísticamente significativas en todas las UPD. Un valor ajustado de $p<0,05$ se consideró significativo en términos estadísticos. Se hizo un análisis cualitativo temático con las preguntas abiertas y las entrevistas personales para investigar y describir las experiencias del personal y los administradores de las UPD, sus percepciones relacionadas con la capacitación, la investigación y la promoción, así como los catalizadores y los obstáculos. Dos autores codificaron todas las respuestas a las preguntas abiertas y las transcripciones de las entrevistas de manera independiente para agrupar los conceptos relacionados e identificar los temas clave. Se determinó la saturación de los temas y se transcribieron citas representativas. Las discrepancias entre los codificadores se resolvieron por consenso.

\section{RESULTADOS}

\section{Características de los centros pediátricos de referencia y las UPD}

Los participantes del programa para las UPD provenían de seis centros pediátricos de referencia y todos recibieron capacitación en AU. Las seis UPD se establecieron en 2010. Al momento de la realización del estudio, en enero de 2018, aún funcionaban cinco UPD. Las UPD se encontraban en cuatro metrópolis con una población de entre 4 y 20 millones; una en Estambul y una en Esmirna, en el norte y oeste de Turquía, respectivamente, y tres en la capital, Ankara, en el centro del país. Estos centros de referencia eran hospitales de niños con una mediana de 271 camas pediátricas (intervalo intercuartílico [IIC]: 142-392) y 60 camas en las unidades de cuidados intensivos neonatales (IIC: 24-105), y prestaban atención ambulatoria a una mediana de 430 niños al día (IIC: 173-790). El espacio destinado por los hospitales para las UPD correspondía a entre una y tres salas, equipadas con instrumentos para las evaluaciones del desarrollo y mobiliario para facilitar la atención centrada en la familia. En tres UPD, había una cámara Gesell; en una, también había equipo de grabación de video. En total, seis pediatras y seis especialistas en desarrollo infantil recibieron capacitación sobre el programa para las UPD. De ellos, cuatro pediatras y dos especialistas en desarrollo infantil aún trabajaban en las UPD. En 2016, el Ministerio de Salud designó a graduados de la capacitación en PDC, una subespecialidad reciente de tres años de duración, para que trabajaran en tres de las UPD.

\section{Servicios clínicos, capacitación e investigación provistos por las UPD}

En total, 23710 niños y sus familias recibieron servicios hospitalarios y ambulatorios en las UPD entre 2011 y 2017. En este último año, se registraron 9085 casos nuevos. Las UPD evaluaron a una mediana de 9 niños y familias por día (IIC: 6-11) y 195 por mes (IIC: 91-204). La mayoría de las derivaciones a las UPD fueron iniciadas por pediatras que trabajaban en los consultorios internos y externos de los hospitales. En la Tabla 2, se muestran los motivos de las derivaciones. Los problemas perinatales fueron el motivo más frecuente e incluyeron a niños derivados porque habían nacido prematuramente, con asfixia u otros problemas y que tenían riesgo de retraso del desarrollo o que ya tenían un retraso del desarrollo debido a causas perinatales. Como se observa en la Tabla 2, la clasificación de las proporciones de los motivos de derivación fue similar en todas las UPD. Cuando se hizo la prueba z para analizar las diferencias en las proporciones en las siete categorías de los motivos de derivaciones de cinco UPD, de los 70 pares resultantes, las proporciones de niños derivados en cada categoría de DD fueron estadística y significativamente diferentes solo en 13 pares $(19 \%)$.

Todas las UPD hicieron una evaluación integral centrada en la familia en el transcurso de un mes desde la derivación del paciente, según la urgencia. La duración de la consulta para la primera evaluación y para el seguimiento fue de 40-60 minutos y de 20-30 minutos, respectivamente. Con mayor frecuencia, los informes de las UPD se hicieron con la GMDI, la GMDI ampliada, las Escalas conductuales 
adaptativas de Vineland y las Escalas Bayley de desarrollo de lactantes y niños pequeños, entre las herramientas provistas en la capacitación.

La capacitación brindada por las UPD a los residentes y otros médicos correspondió a seminarios y cursos sobre DPI y DD, capacitación sobre el uso de la GMDI y sobre derechos y legislaciones relativas a las DD. Se instauró una rotación de un mes en la UPD para los residentes solo en una institución, a pesar de que los cinco centros ofrecían un programa de residencia de cuatro años. En el período de siete años, las UPD llevaron a cabo 14 proyectos de investigación. Las UPD promocionaron una revisión de las legislaciones relativas a la discapacidad, que no diferenciaban a los niños de los adultos, y de la pediatría del desarrollo como subespecialidad. Estos esfuerzos tuvieron un gran alcance y, en 2011, se aprobó la pediatría del desarrollo como subespecialidad en Turquía y se estableció la Asociación Turca de Pediatría del Desarrollo. En 2015, las UPD celebraron el primer Congreso Internacional de Pediatría del Desarrollo en Estambul y se formó la Asociación Internacional de Pediatría del Desarrollo (International Developmental Pediatrics Association, IDPA). En 2019, los esfuerzos de las UPD conllevaron la promulgación de una nueva legislación denominada Legislación de necesidades especiales para niños, que permite la evaluación de los beneficios para niños con discapacidad con un sistema no estigmatizante, basado en las necesidades y la CIF y centrado en la familia.

\section{Resultados del análisis cualitativo de los catalizadores y obstáculos en los servicios de las UPD}

Según los resultados del análisis cualitativo, los catalizadores más importantes del programa fueron la confianza, la motivación y la persistencia de los médicos de las UPD. Los médicos indicaron que su motivación surgió de la idoneidad de la capacitación sobre las UPD y la satisfacción de los niños y las familias a los que atendieron. Los médicos y administradores declararon que los niños con DD y sus familias habían sido derivados al área de neurología o psiquiatría pediátrica previamente, pero no recibían evaluaciones integrales; la gestión de los casos fue posible en las UPD. Otro catalizador fue el respaldo de los administradores de los hospitales, quienes brindaron el espacio y permitieron destinar el tiempo. Los obstáculos fueron problemas con el reembolso y el mantenimiento del personal de las UPD. Algunos de los médicos capacitados fueron trasladados a otros consultorios por la administración debido a las necesidades del hospital. Los administradores informaron que las consultas prolongadas con los pacientes condujeron a una menor cantidad de niños vistos en las UPD en comparación con otros consultorios, lo que derivó en una "pérdida de dinero" para el sistema de salud basado en el rendimiento. Un tema repetitivo fue que los médicos de las UPD sentían la obligación de demostrar la necesidad de los servicios brindados en las UPD en un entorno pediátrico que aún se basaba en el modelo médico y se centraba en la sobrevida de los niños.

\section{DISCUSIÓN}

En este estudio se informan los resultados a largo plazo del programa de capacitación para las UPD en relación con el abordaje del DPI y las DD en Turquía. Según nuestros hallazgos, el programa de capacitación logró la meta de crear UPD sostenibles en centros pediátricos de referencia de envergadura. Siete años después de la capacitación, todas las UPD establecidas, excepto una, aún funcionaban y continuaban brindando servicios a una gran cantidad de niños con diversos riesgos y dificultades del desarrollo.

Los estudios sobre los programas de capacitación para los pediatras sobre conceptos relacionados con el DPI y las DD se llevan a cabo principalmente en los PIA, donde los servicios del desarrollo son un componente de la atención pediátrica de alta calidad. ${ }^{9,23} \mathrm{La}$ investigación relativa a la evaluación de los planes de estudio y los modelos de capacitación específicos de la pediatría está surgiendo en los $\mathrm{PIBM},{ }^{24}$ y el interés por la capacitación de los pediatras en el DPI y las DD es cada vez mayor. ${ }^{11}$ Dos estudios realizados en Nepal demostraron que existen brechas en los conocimientos y las habilidades de los pediatras y se requiere capacitación. ${ }^{25,26}$ Las declaraciones de consenso recientes de India ${ }^{27}$ abordaron la necesidad de brindar capacitación sobre las DD a los pediatras. Dado que la mortalidad en los PIBM ha disminuido ${ }^{4}$ y la comunidad pediátrica se centra en el DPI y las DD, nuestro estudio ofrece información oportuna sobre cómo pueden implementarse los programas de capacitación.

Las implicancias de nuestro estudio atañen a la viabilidad y sostenibilidad del programa para las UPD en Turquía, pero posiblemente también a otros PIBM con sistemas de salud similares. De acuerdo con nuestros resultados, 
la capacitación sobre las UPD, de tres meses de duración, fue viable y se sostuvo tras siete años; todas las UPD, excepto una, aún funcionaban, y la cantidad de niños que recibieron servicios aumentó con el paso del tiempo. Las derivaciones incluyeron niños pequeños con DD, y los problemas perinatales fueron el principal motivo de derivación. Este perfil de derivaciones difiere de los motivos de derivación informados en los servicios del desarrollo de los PIA, donde el perfil incluye autismo, trastorno por déficit de atención con hiperactividad y trastornos de aprendizaje. ${ }^{10}$ Esta diferencia podría deberse a que el programa para las UPD se centró en el DPI, pero también a que se reconocieron los problemas perinatales como la principal causa de morbilidad del desarrollo en los PIBM. Cuatro UPD se encontraban en hospitales universitarios típicos, de gran tamaño y similares, y las diferencias en el porcentaje de niños derivados a las UPD fue, en su mayoría, similar; mientras que la UPD-5 estaba en un hospital materno infantil de referencia, lo que explica la mayor proporción de derivaciones de niños con problemas perinatales. Estos hallazgos indican que podría ser posible que otros centros predigan y planifiquen capacitaciones y servicios que puedan satisfacer las necesidades de la población derivada.

Nuestros resultados sobre la sostenibilidad se alinean con los estudios que destacan la importancia de la selección de los participantes y el personal. ${ }^{28}$ Los médicos seleccionados se ofrecieron voluntariamente para la capacitación, pero no se les había informado cabalmente sobre los salarios menores en función del rendimiento ni se les solicitó un compromiso a largo plazo. La mitad de estos médicos se trasladó a otros puestos. Se recomienda que los candidatos a estos programas de capacitación se elijan entre médicos voluntarios que cuenten con toda la información y que tengan un compromiso a largo plazo con la UPD.

Con los hallazgos del análisis cualitativo sobre los principales catalizadores de los servicios sostenibles es posible volver a enfatizar las recomendaciones del proyecto transcendental denominado North Carolina Assuring Better Child Health and Development Project, que observó que el elemento clave para el éxito era "identificar a un médico cabecilla para que lidere la actividad del proyecto". ${ }^{29}$ Este proyecto también destacó la importancia de "alinear las metas con socios colaboradores para asegurar la participación activa de los socios", y la falta de esto fue el principal obstáculo en los servicios de las UPD.
Las políticas del socio colaborador (el Ministerio de Salud) no estaban en línea con la meta de invertir tiempo y recursos en los niños con DD. Para las iniciativas como el programa para las UPD, es fundamental que los sistemas de salud reconozcan, valoren y paguen los servicios relacionados con el DPI y las DD. Estos programas se fortalecerían si recibieran respaldo a escala de organizaciones internacionales, en particular la OMS, UNICEF y la Asociación Internacional de Pediatría. La perspectiva de parte de los administradores de que los servicios de las UPD son una "pérdida de dinero" requiere atención. Los esfuerzos de promoción ante los ministerios de salud deben centrarse en explicar que los servicios de las UPD se implementan para satisfacer mejor las necesidades de cada niño y familia y que deben verse como una obligación ética y una inversión a futuro del país, no una operación financiera a corto plazo.

La fortaleza más importante de este estudio es que obtuvo resultados a largo plazo del programa de capacitación con métodos cualicuantitativos. La principal limitación fue que no se pudo aplicar una evaluación independiente. Los datos cuantitativos, como la cantidad de pacientes atendidos, no habrían cambiado, pero no es claro si se hubieran notado distintos catalizadores y obstáculos con evaluaciones cualitativas independientes. Otra limitación del estudio fue la falta de datos sobre los motivos de las derivaciones y sobre cómo los niños con DD recibían atención en los centros de referencia antes del establecimiento del programa para las UPD.

\section{CONCLUSIONES}

En esta era, en la que las Naciones Unidas han incorporado el abordaje del DPI a los Objetivos de Desarrollo Sostenible, es sumamente necesario avanzar en el abordaje del DPI y las DD en los PIBM. Se aplicó y evaluó el programa para las UPD en Turquía, que podría aplicarse y adaptarse a las necesidades de otros países que enfrenten desafíos similares en relación con la capacitación en la pediatría del desarrollo.

\section{REFERENCIAS}

1. World Health Organization, United Nations Children's Fund, World Bank Group. Nurturing care for early childhood development: a framework for helping children survive and thrive to transform health and human potential. Geneva: World Health Organization; 2018.

2. Black MM, Walker SP, Fernald LCH, Andersen CT, et al. Early childhood development coming of age: science through the life course. Lancet. 2017; 389(10064):77-90. 
3. Global Research on Developmental Disabilities Collaborators. Developmental disabilities among children younger than 5 years in 195 countries and territories, 19902016: a systematic analysis for the Global Burden of Disease Study 2016. Lancet Glob Health. 2018; 6(10):e1100-21.

4. GBD 2015 Child Mortality Collaborators. Global, regional, national, and selected subnational levels of stillbirths, neonatal, infant, and under-5 mortality, 1980-2015: a systematic analysis for the Global Burden of Disease Study 2015. Lancet. 2016; 388(10053):1725-74.

5. Institute of Medicine (US), Committee on Nervous System Disorders in Developing Countries. Neurological, Psychiatric, and Developmental Disorders: Meeting the Challenge in the Developing World. Washington DC: National Academy Press; 2001.

6. Vaivada T, Gaffey MF, Bhutta ZA. Promoting Early Child Development with Interventions in Health and Nutrition: A Systematic Review. Pediatrics. 2017; 140(2):e20164308.

7. McDowell MJ, Lesslie JM. Long-term outcomes for children with neurodevelopmental disorders: Are they core business for paediatricians? I Paediatr Child Health. 2018; 54(5):469-73.

8. Porter B, Gadassi H, GrossmanZ, KeremE, etal.Community paediatrics in Israel: the 'Goshen' model for change. Arch Dis Child. 2017; 102(9):795-7.

9. Stein RE, Storfer-Isser A, Kerker BD, Garner A, et al. Does length of Developmental Behavioral Pediatrics training matter? Acad Pediatr. 2017; 17(1):61-7.

10. Haggerty RJ, Friedman SB. History of DevelopmentalBehavioral Pediatrics. J Dev Behav Pediatr. 2003; 24(Suppl 1):S1-18.

11. Kraus de Camargo OA. Children are the futuretheir development matters report. First International Developmental Pediatrics Congress. BJMHH. 2016; 4(1): 29-32.

12. World Health Organization. Developmental difficulties in early childhood: prevention, early identification, assessment and intervention in low and middle-income countries: A review. Geneva: WHO; 2012.

13. Ertem IO, Atay G, Dogan DG, Bayhan A, et al. Mothers' knowledge of young child development in a developing country. Child Care Health Dev. 2007; 33(6):728-37.

14. Ertem IO, Pekcici EB, Gok CG, Ozbas S, et al. Addressing early childhood development in primary health care: experience from a middle-income country. J Dev Behav Pediatr. 2009; 30(4):319-26.

15. Mustafayev R, Seyid-Mammadova T, Gulumser O, Balta $\mathrm{H}$, et al. ADD-ECD: A Cascading Package To Address Developmental Difficulties And Early Childhood Development. Book of Abstracts. $2^{\text {nd }}$ International Developmental Pediatrics Association Congress. 7-10 Dec 2017. Mumbai, India. 2017:13.

16. Ertem IO, Dogan DG, Gok CG, Kizilates SU, et al. A guide for monitoring child development in low- and middleincome countries. Pediatrics. 2008; 121(3):e581-9.
17. Ertem IO, Krishnamurthy V, Mulaudzi MC, Sguassero $Y$, et al. Similarities and differences in child development from birth to age 3 years by sex and across four countries: a cross-sectional, observational study. Lancet Glob Health. 2018; 6(3):e279-91.

18. Ertem IO, Krishnamurthy V, Mulaudzi MC, Sguassero Y, et al. Validation of the International Guide for Monitoring Child Development demonstrates good sensitivity and specificity in four diverse countries. Acta Paediatr. 2019; 108(6):1074-86.

19. BronfenbrennerU,CeciSJ.Nature-nurture reconceptualized in developmental perspective: A bioecological model. Psychol Rev. 1994; 101(4):568-86.

20. Committee on Hospital Care and Institute for Patient- And Family-Centered Care. Patient- and family-centered care and the pediatrician's role. Pediatrics.2012; 129(2):394-404.

21. World Health Organization. International Classification of Functioning, Disability and Health (ICF). Geneva: WHO; 2001.

22. Bell A, Corfield M, Davies J, Richardson N. Collaborative transdisciplinary intervention in early years- putting theory into practice. Child Care Health Dev. 2010; 36(1):142-8.

23. Kuo AA, Inkelas M, Maidenberg M, Lotstein DS, et al. Pediatricians' roles in the provision of developmental services: an international study. J Dev Behav Pediatr. 2009; 30(4):331-9.

24. Ganapathi L, Martins Y, Schumann D, Russ C. Overcoming challenges to develop pediatric postgraduate training programs in low- and middle-income countries. Educ Health (Abingdon). 2014; 27(3):277-82.

25. Shrestha M, Shrestha R. Symptom recognition to diagnosis of autism in Nepal. J Autism Dev Disord. 2014; 44(6):1483-5.

26. Rimal HS, Pokharel A, Saha V. Study on developmentalbehavioural pediatrics training experiences of pediatricians and pediatric trainees working in Nepal. Kathmandu Univ Med J (KUMJ). 2013; 11(43):191-5.

27. National Consultation Meeting for developing Indian Academy of Pediatrics (IAP), Guidelines on NeurodevelopmentalDisorders under the aegis of IAP Childhood Disability Group and the Committee on Child Development and Neurodevelopmental Disorders, C Nair MK, Prasad C, et al. Consensus Statement of the Indian Academy of Pediatrics on Evaluation and Management of Learning Disability. Indian Pediatr. 2017; 54(7):574-80.

28. Bassett ML, Ramsey WP, Chan CC. Improving medical personnel selection and appointment processes. Int J Health Care Qual Assur. 2012; 25(5):442-52.

29. Earls MF, Hay SS. Setting the stage for success: implementation of developmental and behavioral screening and surveillance in primary care practice-the North Carolina Assuring Better Child Health and Development (ABCD) Project. Pediatrics. 2006; 118(1):e183-8. 